\title{
Research Square \\ Cardiogenic shock treated with \\ immunosuppression: a case presentation of acute fulminant eosinophilic myocarditis
}

Nikhil Chatrath ( $\square$ nikhil.chatrath@doctors.org.uk)

Royal Brompton and Harefield NHS Trust: Royal Brompton and Harefield NHS Foundation Trust https://orcid.org/0000-0001-5620-6049

Owais Dar

Royal Brompton and Harefield NHS Trust: Royal Brompton and Harefield NHS Foundation Trust

\section{Research Article}

Keywords: Eosinophilic Myocarditis, Ulcerative Colitis, Immunosuppression, Outcomes

Posted Date: August 9th, 2021

DOl: https://doi.org/10.21203/rs.3.rs-296128/v1

License: (c) (i) This work is licensed under a Creative Commons Attribution 4.0 International License.

Read Full License 


\section{Abstract}

Eosinophilic Myocarditis is a rapidly progressive form of myocarditis, associated with several underlying conditions including inflammatory bowel disease. A 34-year old male with a history of Ulcerative Colitis on Infliximab presented with chest pain, dyspnoea and a recent febrile illness. Serum troponin levels were raised and ECG showed right bundle brunch block. Subsequent emergency coronary angiography showed unobstructed coronary arteries but echocardiography revealed severely impaired left ventricular function. He clinically deteriorated rapidly, developing atrio-ventricular block and cardiogenic shock requiring inotropic and mechanical circulatory support. Endomyocardial biopsy specimens showed diffuse eosinophilic infiltration. Aggressive corticosteroid therapy was initiated and there was a gradual resolution of ventricular function. Peripheral eosinophil counts were within the normal range throughout the illness. Disease activity was monitored with multimodality imaging including PET-CT. Immunosuppression was maintained with Ciclosporin and Mycophenolate Mofetil, avoiding long-term steroid therapy.

\section{Case Description}

A 34-year old male presented with a 2 day history of intermittent chest pain and dyspnoea with generalised malaise and a febrile illness 5 days prior. He had a history of Ulcerative Colitis (UC) on Infliximab, having previously developed pancreatitis secondary to Azathioprine. He had no risk factors for cardiovascular disease.

On admission, the ECG showed new right bundle branch block (RBBB) and bloods revealed a high-

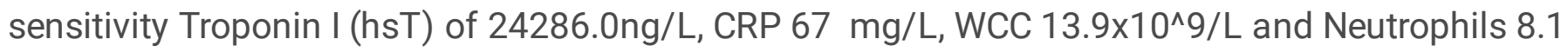
10^9/L. Transthoracic echocardiography (TTE) revealed a non-dilated left ventricle with severe systolic dysfunction (LVSD), an estimated ejection fraction (EF) of $20 \%$ and a mild pericardial effusion. Emergency coronary angiography showed unobstructed coronary arteries and an initial attempt at right ventricular endomyocardial biopsy (EMB) was complicated by haemodynamically compromised complete heart block requiring temporary pacemaker insertion.

He was transferred to the intensive care unit (ICU) due to ongoing haemodynamic instability and a fever of 38.7, commenced on intravenous furosemide, milrinone and Co-Amoxiclav. The working diagnosis was of a fulminant myocarditis. Further deterioration evidenced by a cardiac index of $1.71 / \mathrm{min} / \mathrm{m} 2$, rising lactate and escalating inotropic requirements resulted in the insertion of an Impella $\mathrm{CP} \circledast$ and a successful EMB. This showed florid myocarditis with diffuse infiltration of predominantly lymphocytes and eosinophils, confirming the diagnosis of Eosinophilic Myocarditis (EM). (See Figure 1).

He was commenced on $1 \mathrm{~g}$ IV Methylprednisolone for 3 days followed by oral prednisolone $(1 \mathrm{mg} / \mathrm{kg}) \mathrm{but}$ needed 3 further days of intravenous methylprednisolone $500 \mathrm{mg}$ due to a rising Troponin and lactate while switching to oral steroids. He was maintained on Hydrocortisone 80mg QDS and Mycophenolate Mofetil (MMF) 500mg BD. 
The inotropes were successfully weaned and Impella explanted 7 days after insertion. A repeat TTE showed an improvement in LV function with an EF of $60 \%$. Cardiac MRI showed global myocardial oedema and limited mid-wall fibrosis compatible with an acute myocarditis. The MMF dose was increased and His Hs-T began to persistently downtrend. He was discharged on Bisoprolol 1.25mg OD, Ramipril 5mg OD, MMF 1.5g BD, Spironolactone 25mg OD and Prednisolone 90mg OD to be reduced by $5 \mathrm{mg}$ per week.

An 18FDG PET-CT scan showed increased FDG uptake in the myocardium involving the septum, anterior and mainly lateral walls suggesting active inflammation (Figure 2a). Over the coming months, he was maintained on MMF $1.5 \mathrm{mg}$ BD with a tapering regime of prednisolone with the addition of Ciclosporin $100 \mathrm{mg}$ BD due to a mildly raised Troponin. After 5 months of treatment, a repeat 18FDG PET revealed the myocardial inflammation had completely resolved with no new areas of active inflammation (Figure $2 b$ ).

He remains stable on MMF and Ciclosporin.

\section{Discussion}

EM is a rare, rapidly progressing form of myocarditis characterised by marked eosinophilic infiltration and inflammation of the myocardium causing myocyte necrosis and endomyocardial fibrosis [1]. It may be associated with hypersensitivity reactions, intercurrent infection, malignancy or immune-mediated disorders, such as eosinophilic granulomatosis with polyangiitis (EGPA) or as part of a hypereosinophilic syndrome (HES) [2,3].

EM associated with Ulcerative Colitis (UC) has previously been described in the literature and is associated with more fulminant forms of myocarditis [4]. Myocarditis related to Infliximab use has also been described, due to a hypersensitivity reaction, but this as the cause of myocarditis in this patient is unlikely given the absence of peripheral eosinophilia [5].

Though the vast majority (over 75\%) of cases of EM have marked peripheral eosinophilia, particularly when associated with inflammatory bowel disease or hypersensitivity reactions, normal eosinophil counts in EM have rarely been described [6,7].

The clinical presentation and severity of EM can vary. According to a systematic review, patients with EM who develop arrythmias in the acute phase, tend to be ventricular tachycardia or fibrillation [8]. High grade atrio-ventricular block, as seen in this patient, has yet to be described and may be suggestive of advanced infiltration of the conduction pathways, reflective of the severity of his disease.

EM can rapidly cause cardiogenic shock and temporary mechanical support may be indicated in a minority of patients [8]. Despite advanced acute heart failure therapies and immunosuppression, the risk of in-hospital mortality remains high, thus prompt diagnosis and treatment are crucial to prevent adverse outcomes [9]. 
Currently, there is a lack of consensus regarding immunosuppression in these patients, particularly as maintenance therapy. There is evidence for the efficacy of corticosteroid therapy, and currently remain the basis of treatment for the majority of patients with EM. However, the duration of treatment and regimen for long-term immunosuppression remains controversial [10].

In this case, the patient had previously developed pancreatitis related to Azathioprine use. Therefore, the choice of long-term immunosuppression that would prevent relapse of UC and recurrence of EM, while avoiding the deleterious effects of long-term corticosteroid therapy, required careful consideration.

Crucially, peripheral eosinophil count was not a reliable marker of adequate immunosuppression, having been within the normal range throughout his illness. This case highlights the role of using other tests such as circulating Troponin levels and evidence of myocardial inflammation on serial PET-CT imaging, as markers to reflect the degree of disease activity and thus adequacy of immunosuppressive therapy.

To our knowledge, this is the first case reported in the literature of EM associated with UC in the absence of peripheral eosinophilia. This patient was maintained on long term immunosuppression with Ciclosporin and MMF, thereby avoiding long-term steroid use.

This case highlights the importance of prompt EMB in these patients to guide immunosuppression, the role of mechanical circulatory support to aid myocardial recovery, and the role of multi-modality imaging including TTE, CMR and PET-CT to assess for improvement in cardiac function and to monitor for response to treatment.

$\mathrm{EM}$ is a rare condition with potentially fatal consequences. The risk of EM recurrence, development of dilated cardiomyopathy or ventricular arrythmias is unknown. Given the lack of clinical trials for the treatment of EM, it is by encouraging the reporting and follow-up of cases of EM that a consensus can be made on the treatment and risk-stratification of these patients.

\section{Declarations}

\section{Acknowledgements}

Dr Alexandra Rice - Consultant Histopathologist, Royal Brompton Hospital

Dr Angelica Barrenechea - Consultant Radiologist, Royal Brompton Hospital

Funding This article was not financially supported by any grant, funding source, or commercial interest.

\section{Conflicts of interest/Competing interests}

Drs. Chatrath and Dar have no conflicts of interest or financial ties to disclose.

\section{Ethics approval}


This study has been approved by local ethics committee and been performed in accordance with the ethical standards laid down in the 1964 Declaration of Helsinki and its later amendments.

\section{Consent for participation and publication}

The patient involved in the case study has provided informed consent for their participation in the study and publication of the case report, including images of histology slides and PET-CT imaging.

\section{Availability of data and material}

Not applicable

\section{Code availability}

Not applicable

\section{Authors' contributions}

The listed authors (Dr. Chatrath and Dr.Dar) were both involved in the conception of the case report, acquisition of the data, drafted the work and revised it critically before approving the submitted version. Both authors agree to be accountable for all aspects of the work.

The participant has consented to the submission of the case report to the journal.

\section{References}

1. Ginsberg F, Parrillo JE. Eosinophilic myocarditis. Heart Fail Clin. 2005;1:419-29.

2. Ammirati $E$, Cipriani M, Musca F, et al. A life-threatening presentation of eosinophilic granulomatosis with polyangiitis. J Cardiovasc Med (Hagerstown). 2016;S2:e109.

3. Roehrl MH, Alexander MP, Hammond SB, Ruzinova M, Wang JY. C.J. O'Hara. Eosinophilic myocarditis in hypereosinophilic syndrome. Am J Hematol. 2011;86:607-8.

4. Murphy K. Waldo O, Lohrmann GM. Tazelaar HD, Jokerst CE. Mookadam F. Eosinophilia and ulcerative colitis associated with eosinophilic myocarditis. Tex Heart Inst J 44: 219-22, 2017).

5. Slattery E, Ismail N, Sheridan J, Eustace K, Harewood G, Patchett S. Myocarditis associated with infliximab: a case report and review of the literature. Inflamm Bowel Dis. 2011 Jul;17(7):1633-4. doi:10.1002/ibd.21546. Epub 2010 Oct 25. PMID: 21674722.

6. Morimoto S. Kubo N, Hiramitsu S. Uemura A, Ohtsuki M. Kato S, Kato Y. Sugiura A, Miyagishima K. Mori N, Yoshida Y. Hishida H. Changes in the peripheral eosinophil count in patients with acute eosinophilic myocarditis. Heart Vessels. 2003; 18:193-196.

7. Fozing T. Zouri N, Tost A, et al Management of a patient with eosinophilic myocarditis and normal peripheral eosinophil count: case report and literature review. Circ Heart Fail 2014;7:692-4. 
8. Brambatti M, Matassini MV, Adler ED, Klingel K, Camici PG, Ammirati E. Eosinophilic Myocarditis: Characteristics, Treatment, and Outcomes. J Am Coll Cardiol. 2017 Nov 7;70(19):2363-2375. doi: 10.1016/j.jacc.2017.09.023. PMID: 29096807.

9. Ammirati E. Cipriani M, Lilliu M, et al Survival and left ventricular function changes in fulminant versus non-fulminant acute myocarditis. Circulation 2017;136:529-45.).

10. (Caforio AL. Pankuweit S, Arbustini E, et alCurrent state of knowledge on aetiology, diagnosis, management, and therapy of myocarditis: a position statement of the European Society of Cardiology Working Group on Myocardial and Pericardial Diseases. Eur Heart J 2013;34: 2636-48, $2648 a-d$.

\section{Figures}
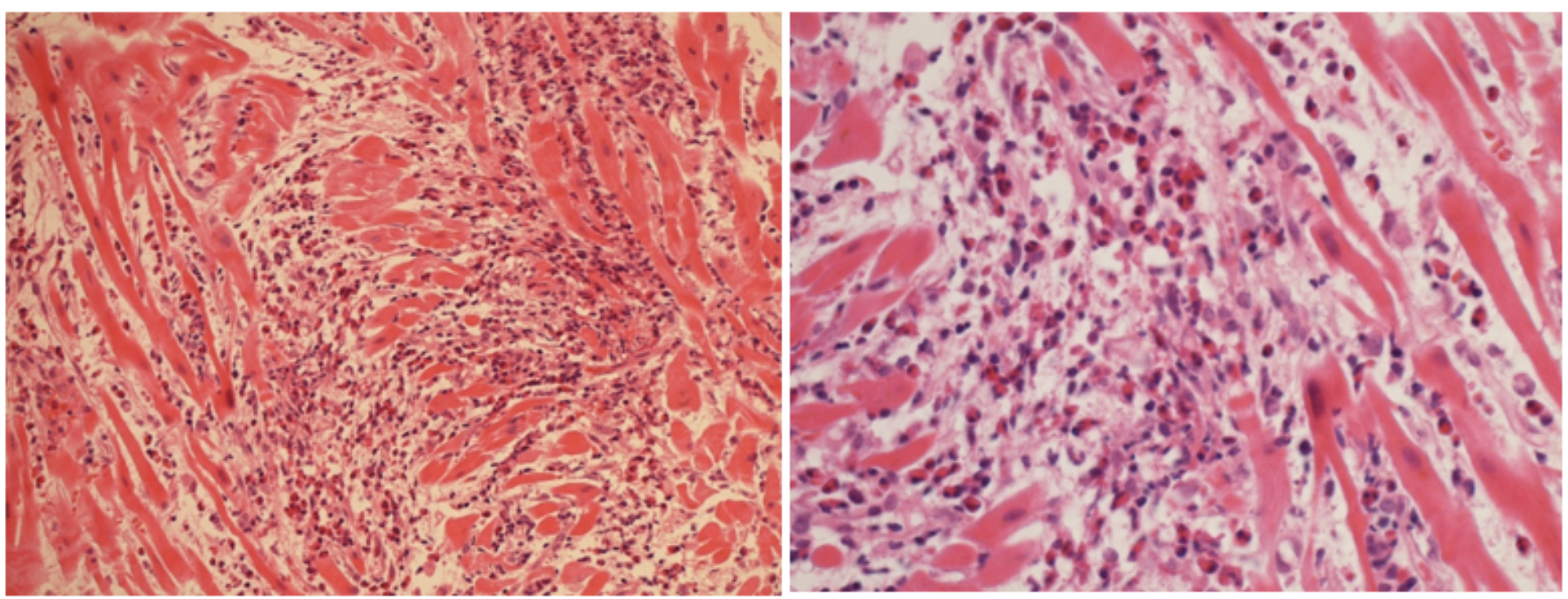

\section{Figure 1}

Eosinophilic infiltration in myocardium with myocyte necrosis (H\&E stain x 200 and x400 magnification). 


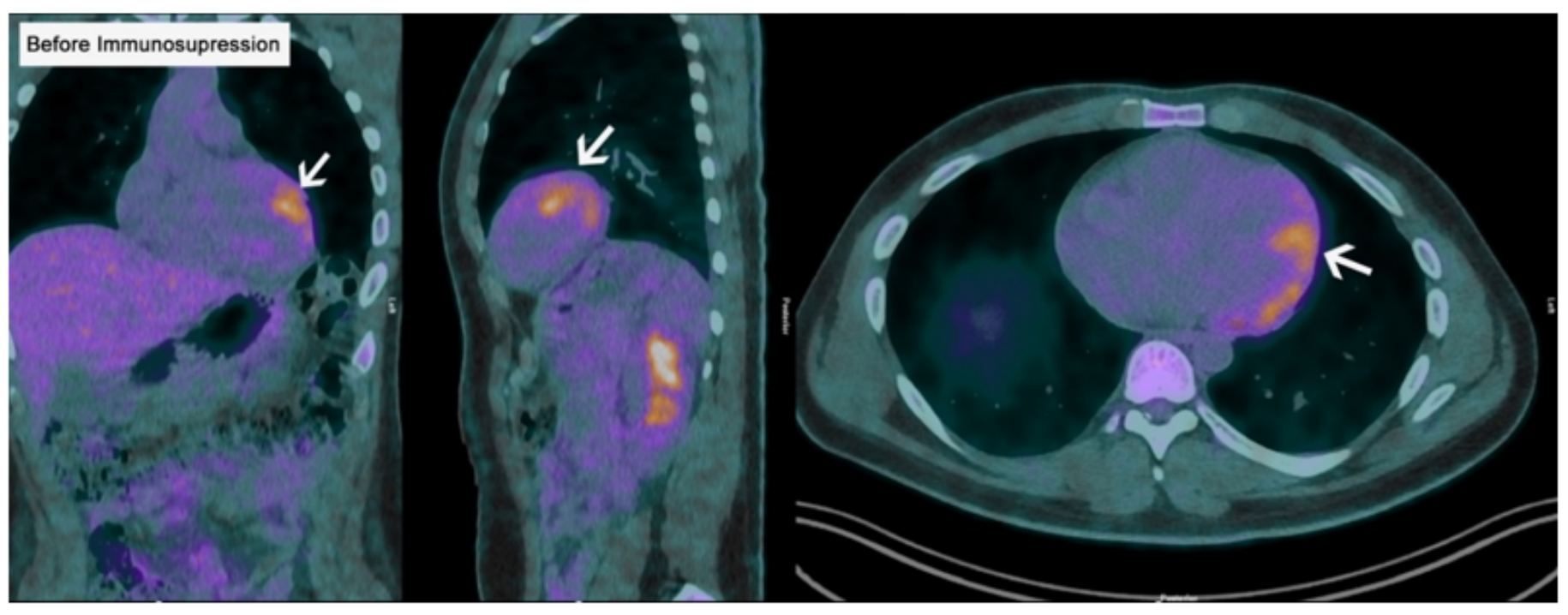

A

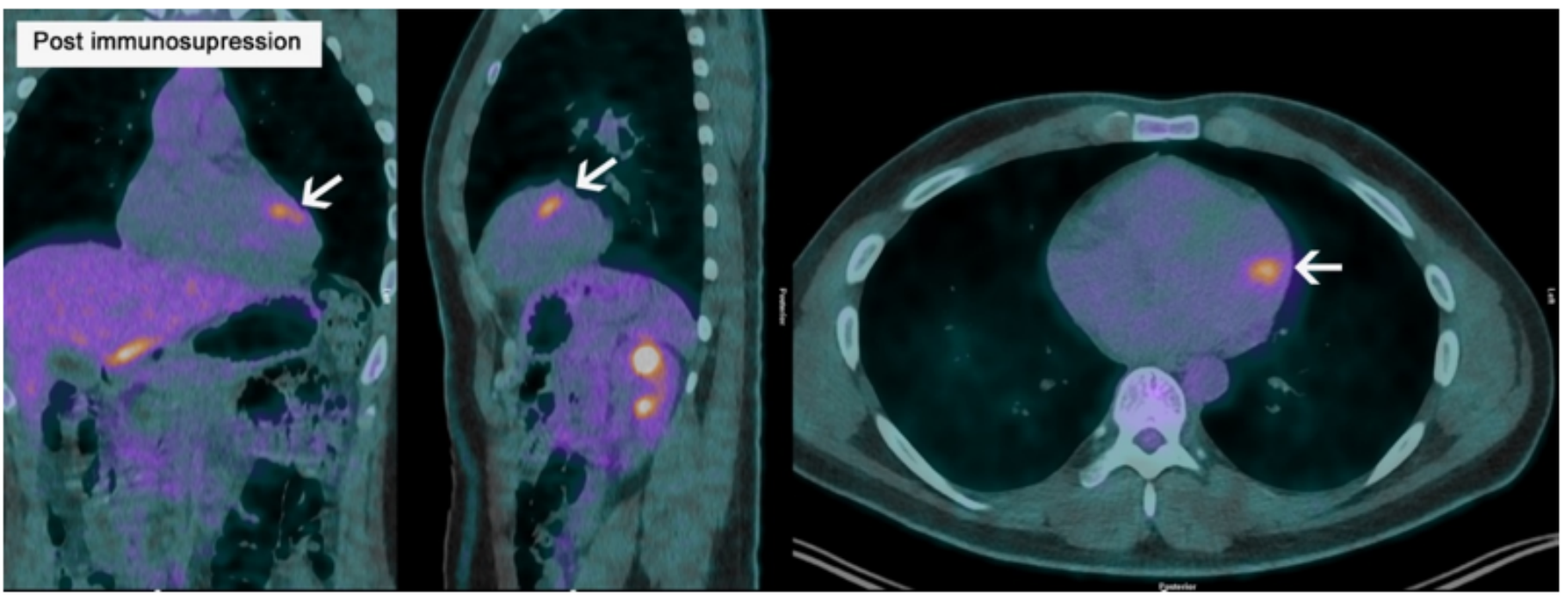

Figure 2

a - PET-CT images using 18F-FDG, showing increased FDG uptake (likely representing inflammation) mainly in the anterolateral wall of the myocardium and the anterolateral papillary muscle (arrows). b PET-CT images using 18F-FDG showing almost complete resolution of FDG uptake along the anterolateral wall of the myocardium and with only FDG uptake persisting in the anterolateral papillary muscle (arrows). 